\title{
Entropy Measures in Finance and Risk Neutral Densities
}

\author{
Muhammad Sheraz ${ }^{1, *}$, Vasile Preda ${ }^{2}$ \\ 1 Department of Financial Engineering Faculty of Science Ningbo University,818 Fenghua Road \\ Ningbo city, Zhejiang Province, China ; E-Mails: muhammad_sheraz84@hotmail.com \\ 2 Faculty of Mathematics and Computer Science University of Bucharest; Str. Academiei nr.14, \\ sector 1, C.P. 010014, Bucharest, Romania.; vasilepreda0@gmail.com \\ * Author to whom correspondence should be addressed; E-Mail: uhammad_sheraz84@hotmail.com; \\ Tel.: +8618312985503
}

Published: 13 November 2015

\begin{abstract}
The application of entropy in finance can be regarded as the extension of the information entropy and the probability entropy. It can be an important tool in various financial methods such as measure of risk, portfolio selection, option pricing and asset pricing. A typical example for the field of option pricing is the Entropy Pricing Theory (EPT) introduced by Les Gulko [1996]. The Black-Scholes model [1973] exhibits the idea of no arbitrage which implies the existence of universal risk-neutral probabilities but unfortunately it does not guarantees the uniqueness of the risk-neutral probabilities. In a second step the parameterization of these risk-neutral probabilities needs a frame of stochastic calculus and to be more specific for example the Black and Scholes frame is controlled by Geometric Brownian Motion (GBM). This implies the existence of riskneutral probabilities in the field of option pricing and their uniqueness is vital. The Shannon entropy can be used in particular manners to evaluate entropy of probability density distribution around some points but in the case of specific events for example deviation from mean and any sudden news for stock returns up (down), needs additional information and this concept of entropy can be generalized. If we want to compare entropy of two distributions by considering the two events i.e. deviation from mean and sudden news then Shannon entropy [1964] assumes implicit certain exchange that occurs as a compromise between contributions from the tail and main mass of the distribution. This is important now to control this trade-off explicitly. In order to solve this problem the use of entropy measures that depend on powers of probability for example Tsallis [1988], Kaniadakis [2001], Ubriaco [2009], Shafee [2007] and Rényi [1961] provide such control. In this article we use entropy measures depend on the powers of the probability. We
\end{abstract}


propose some entropy maximization problems in order to obtain the risk neutral densities.

We present also the European call and put in this frame work.

Keywords: Entropy Measures; Risk Neutral Densities; Black-Scholes Option Pricing

PACS Codes: 89.65.-s Social and economic systems

\section{Introduction}

The application of entropy in Finance can be regarded as the extension of both information entropy and probability entropy. Since last two decades the application of entropy measures in finance has become a very important tool for the methods of portfolio selection and asset pricing. The famous Black-Scholes model [1] assumes the condition of no arbitrage which implies the universe of riskneutral probabilities. The uniqueness of these risk-neutral probabilities is very crucial. The stock price process is controlled by Geometric Brownian Motion (GBM) in Black and Scholes model and in this framework stochastic calculus is vital. The Entropy Pricing Theory (EPT) was introduced by Les Gulko as an alternative method for the construction of risk-neutral probabilities without relying on stochastic calculus $[8,9,10]$. The Principle of Maximum Entropy (MEP) was used to estimate the distribution of an asset from a set of option prices [13]. Beside this work the maximum entropy principle was used to retrieve the risk-neutral density of future stock risks or other asset risks [21]. The Renyi entropy [20] generalizes the frequently used Shannon entropy [22] and it has been used for option price calibration [6]. Some more extensions for the use of entropy for the case of random homogeneous systems with complete connections can be found in [12]. Recently Preda et al. used Tsallis and Kaniadakis entropy measures for the case of semi-Markov regime switching interest rate models [17]. Preda et al. have also introduced the new classes of Lorenz curves by maximizing Tsallis entropy under mean and Gini's equality and inequality constraints [18]. For maximum entropy distribution of asset returns, application of entropy in finance, entropy maximization problems, and others can be found in $[3,7,13,15,16,26]$.

In this article we use three different types of entropy measures to find risk-neutral densities using the framework of EPT for stock options [20,23,24]. In Section 2 we present the introduction of EPT and formulation of our problems, and then we further develop the structure to obtain our new results. We discuss the approach of Lambert function to get risk-neutral density of stock options then we present a new approach. We introduce the weighted entropy maximization problems and the expected utility-weighted entropy (EU-WE) framework. We extend our results for pricing European call and put options. Section 3 concludes our results.

\section{Results and Discussion}

We use the concept of EPT $[8,9,10]$.The term market belief is vital in option pricing and the current price of any risky asset indicates this belief. The future picture of the market up (down) reflects a state of maximum possible uncertainty; therefore market belief for the future performance of an efficient 
price is characterized by maximum uncertainty. Consider a risky asset on time interval[0,T] . Let $Y_{T}$ be asset price process of $\mathrm{S}_{T}$ at future time $T, G$ as state space, a subset of real line $R, \mathrm{~g}\left(\mathrm{~S}_{T}\right)$ the probability densities on $\mathrm{P}, f\left(S_{T}\right)$ efficient market belief and $H(g)$ the index of market uncertainty about $Y_{T}$. The $H(g)$ is defined on the set of beliefs $g\left(S_{T}\right)$ therefore the efficient market belief $f\left(S_{T}\right)$ maximizes $H(g)$.

We can determine $f\left(S_{T}\right)$ given $H(g)$ with some relevant information about current price of $S$. The index of the market uncertainty about $Y_{T}$ as a Shannon entropy can be written as:

$$
H(g)=-E^{g}\left[\ln \mathrm{g}\left(Y_{T}\right)\right]
$$

In the above equation $H(g)$ is a functional defined on $g\left(S_{T}\right), f\left(S_{T}\right)$ which maximize $H(g)$ is called the entropy of random variable $Y_{T}$ and used to measure the degree of uncertainty of $g\left(S_{T}\right)$. The maximum entropy characterizes the market beliefs regardless of the subjective risk preferences and it is useful to find the risk neutral beliefs in incomplete arbitrage free markets. The maximum entropy market belief $f\left(S_{T}\right)$ as a solution to the maximum entropy problem can be written as follows:

$$
f=\arg \max \{H(g), g \in G\}
$$

\subsection{Shafee Entropy Measure and Risk Neutral Densities}

In 2007 non additive entropy was proposed [23]. It gives the general form that is non-extensive like Tsallis, but is linearly dependent on component entropies. The mathematical description of the new entropy functions was in discrete case and for our problem we consider the continuous cases as an analogue of the discrete case:

$$
H_{q}(g)=-E^{g}\left[g\left(Y_{T}\right)^{q-1} \ln \mathrm{g}\left(Y_{T}\right)\right], q>0, q \neq 1
$$

The Lambert function has become an important tool since its beginning with Lambert-1768 and Euler in 1779. The Lambert function $W$ is a multi-valued complex function which is defined as the solution of the equation where $W(z) e^{W(z)}=z, z$ is a complex number. If $z$ is a real number such that, $z \geq-e^{-1}$, then $W(z)$ becomes a real function with two possible real branches taking values in $(-\infty,-1]$ and $[-1, \infty) \cdot[13]$.

Lemma 2.2. [13] Let $a, b$ and $c$ be any fixed complex numbers. Then solution of equation $z+a b^{z}=c$

with $z \in C$ is, $z=c-\frac{1}{\log (b)} W\left(a b^{c} \log (b)\right)$, where $W$ is Lambert function and $\mathrm{C}$ denotes complex numbers.

Preda and Sheraz have recently studied Shafee entropy maximization problems for the case of risk neutral densities. They have obtained solutions using the Lambert function and a new approach for various cases such as Expected Utility-Weighted Shafee Entropy (EU-WSE) frame work [19].

Theorem 2.1. Consider the following entropy maximization problem:

$$
\max -E^{g}\left[u\left(Y_{T}\right) g^{q-1}\left(Y_{T}\right) \ln \mathrm{g}\left(Y_{T}\right)\right]
$$

Subjet to 


$$
\begin{array}{ll}
E^{g}\left[I_{\left\{Y_{T}>0\right\}}\right]=1 & \mathrm{C}-1 \\
E^{g}\left[u\left(Y_{T}\right) \varphi_{i}\left(Y_{T}\right)\right]=a_{i}, i=\overline{1, n}, & \mathrm{C}-2
\end{array}
$$

where $u\left(S_{T}\right)>0, \varphi_{i}: R \rightarrow R$ and $a_{1}, \ldots a_{n}$ are given real values. The solution of the problem is

$$
\mathrm{g}\left(S_{T}\right)=\left[\frac{q u\left(S_{T}\right) W\left(\frac{\left(\alpha+\sum_{i=1}^{n} \beta_{i} u\left(S_{T}\right) \varphi_{i}\left(S_{T}\right)\right)(1-q)}{q u\left(S_{T}\right)} \exp \left\{-\left(\frac{1-q}{q}\right)\right\}\right)}{\left(\alpha+\sum_{i=1}^{n} \beta_{i} u\left(S_{T}\right) \varphi_{i}\left(S_{T}\right)\right)(1-q)}\right]^{\frac{1}{1-q}}
$$

where $\alpha, \beta_{1}, \ldots \beta_{n}$ are Langrage multipliers, and $\mathrm{W}$ is the Lambert function.

\subsection{Ubriaco Entropy Measure}

Ubriaco (2009) proposed a new entropy measure based on fractional calculus and showed this new entropy has the same properties as Shannon entropy except additivity. We consider the analogue of the discrete case of non additive entropy defined by [24]. We suppose that all expectations are also well defined and underlying optimization problems admit solutions for some continuous cases. More details in $[2,5,15]$. We may write for the discrete case as:

$$
H(p)=\sum_{i=1}^{n} p_{i}\left(\ln \left(\frac{1}{p_{i}}\right)\right)^{d}
$$

where $H(p)=\sum_{i=1}^{n} H_{i}\left(p_{i}\right)$ is positive and by using condition $\frac{\partial H}{\partial p_{i}}=0$ and $H_{i}\left(p_{i}\right)=p_{i}\left(\ln \left(\frac{1}{p_{i}}\right)\right)^{d}$. Also $H_{i}\left(p_{i}\right)$ has maximum at $p_{i}=e^{-d}$ and with a second derivative at this point, $\frac{\partial^{2} H_{i}}{\partial p_{i}^{2}}=-d^{d-1} e^{d}$. Similarly in the continuous case if $g\left(S_{T}\right)$ is the density function we may write as follows:

$$
H\left(\mathrm{~g}\left(S_{T}\right)\right)=E^{g}\left(\ln \left(\frac{1}{g\left(S_{T}\right)}\right)\right)^{d}, 0<g \leq 1
$$

We obtain the Shannon entropy for $d=1$.

Lemma 2.1. $\psi=x\left(\ln \frac{1}{x}\right)^{d}, 0<x \leq 1, d>0, \psi^{\prime}:\left(0, e^{-d}\right) \rightarrow(0, \infty)$ then $\psi^{\prime}$ the first derivative of $\psi$ admits inverse. 
$\psi^{\prime}$ is strictly increasing therefore the proof of Lemma 2.1 is obvious.

Theorem 2.2. Consider the following maximum entropy problem for the case of Ubriaco entropy measure:

$$
\max H(g)=E^{g}\left[\left(\ln \left(\frac{1}{g\left(Y_{T}\right)}\right)\right)^{d}\right]
$$

Subject to

$$
\begin{array}{ll}
E^{g}\left[I_{\left\{Y_{T}>0\right\}}\right]=1 & \mathrm{C}-1 \\
E^{g}\left[Y_{T}\right]=S_{0} e^{r T} & \mathrm{C}-2 \\
E^{g}\left[\left(Y_{T}-K_{0}\right)^{+}\right]=C_{0} e^{r T} & \mathrm{C}-3
\end{array}
$$

where $K_{0}$ is the strike price, $T$ is time to expiry and $r$ is the risk free interest rate. Then the risk neutral density $g\left(S_{T}\right)$ is given by:

$$
g\left(S_{T}\right)=\left(\psi^{\prime}\right)^{-1}\left(\lambda+\beta_{1} S_{T}+\beta_{2}\left(S_{T}-K_{0}\right)^{+}\right)
$$

where $\lambda, \beta_{1}$ and $\beta_{2}$ are Lagrange multipliers and need to be determined by $\mathrm{C}-1, \mathrm{C}-2$ and $\mathrm{C}-3$.

Proof. We can proof the above result by using the calculus of variations for optimization of functionals (see Luenberger -1969, Borwein -2003). The corresponding Lagrangian can be written as:

$$
L(g, \lambda)=E^{g}\left[\left(\ln \left(\frac{1}{g\left(Y_{T}\right)}\right)\right)^{d}\right]-\lambda_{1}\left(E^{g}\left[I_{\left\{Y_{T}>0\right\}}\right]-1\right) \beta_{1}\left(E^{g}\left[Y_{T}\right]-S_{0} e^{r T}\right)-\beta_{2}\left(E^{g}\left(S_{T}-K_{0}\right)^{+}-C_{0} e^{r T}\right)
$$

Then $g$ has to satisfy the relation:

$$
\left(\ln \left(\frac{1}{g\left(S_{T}\right)}\right)\right)^{d}-d\left(\ln \left(\frac{1}{g\left(S_{T}\right)}\right)\right)^{d-1}-\lambda_{1}-\beta_{1} S_{T}-\beta_{2}\left(S_{T}-K_{0}\right)^{+}=0
$$

Using Lemma 2.1.1 we can write the above equation:

$$
\left(\ln \left(\frac{1}{g\left(S_{T}\right)}\right)\right)^{d}-d\left(\ln \left(\frac{1}{g\left(S_{T}\right)}\right)\right)^{d-1}=\psi^{\prime}\left(g\left(S_{T}\right)\right)
$$

Therefore,

$$
\begin{array}{ll} 
& \psi^{\prime}\left(g\left(S_{T}\right)\right)=\lambda_{1}+\beta_{1} S_{T}+\beta_{2}\left(S_{T}-K_{0}\right)^{+} \\
& g\left(S_{T}\right)=\left(\psi^{\prime}\right)^{-1}\left(\lambda_{1}+\beta_{1} S_{T}+\beta_{2}\left(S_{T}-K_{0}\right)^{+}\right)
\end{array}
$$

The proof is complete. 
We can extend the previous result of theorem 2.2 for the case of weighted entropy. The weighted entropy was first defined by Guiasu [11], considering the two basic concepts of objective probability and subjective utility. If $u\left(S_{T}\right)>0$ then solution of the weighted Ubriaco entropy maximization problem subject to the constraints $\mathrm{C}-1, \mathrm{C}-2$ and $\mathrm{C}-3$ is given by:

$$
g\left(S_{T}\right)=\frac{\left(\psi^{\prime}\right)^{-1}\left(\lambda_{1}+\beta_{1} S_{T}+\beta_{2}\left(S_{T}-K_{0}\right)^{+}\right)}{u\left(S_{T}\right)}
$$

\subsection{Rényi Entropy Measure}

The Rényi entropy is named after Alfréd Rényi and in the field of information theory the Rényi entropy generalizes, the Shannon entropy. The relative entropy minimization has been used extensively in the past for the calibration of the financial models. In financial modeling most of the entropy based calibration methods depend on the use of the logarithmic entropy measure of Shannon. A one parameter family of entropies generalizing the logarithmic entropy measure of Shannon and Wiener was considered by Rényi [20,22]. In 2007 Dorje et al. [6] have studied the case of Rényi entropy for calibration of option pricing and explained a piece of information that one drawback in the use of logarithmic entropy measures is that if the only source of information used to maximize entropy are the market prices for the vanilla option, then the resulting density function is necessarily of exponential form. The use of Rényi entropy is the more general case for the calibration of risk neutral price distribution.

The Rényi entropy of order $r$ where $r \geq 0, r \neq 1$ is defined as:

$$
H_{r}(X)=\frac{1}{1-r} \log \left(\sum_{i=1}^{n} g_{i}^{r}(X)\right)
$$

where $X$ is a discrete random variable and $p_{i}=\operatorname{Pr}(X=i)$ for $i=1,2, \ldots, n$ and $r \rightarrow 0$ the Rényi entropy is just the logarithm of the size of the support of $X$ and for $r \rightarrow 1$ equals to Shannon's entropy. In the case of continuous random variable we may write Rényi entropy as:

$$
H_{r}(X)=\frac{1}{1-r} \log E^{g}\left(g^{r-1}(X)\right)
$$

We extend our results for the risk-neutral densities in the case of Rényi entropy. In this Section we present our main results for Rényi entropy. Also we present pricing of European call and put options. We suppose that all expectations are also well defined and underlying optimization problems admit solutions for some continuous cases, where $E^{g}$ is expectation relative to probability density $g\left(S_{T}\right)$ and $S_{T}$ is asset price at time $T$.

Theorem 2.3. Consider the Rényi entropy maximization problem:

$$
\max H_{r}(g)=\frac{1}{1-r} \ln E^{g}\left(g^{r-1}\left(Y_{T}\right)\right)
$$

subject to 


$$
\begin{gathered}
E^{g}\left[I_{\left\{-\infty<Y_{T}<\infty\right\}}\right]=1 \quad \mathrm{C}-1 \\
E^{g}\left[\varphi_{i}\left(Y_{T}\right)\right]=c_{i}, i=\overline{1, n}, \quad \mathrm{C}-2
\end{gathered}
$$

where, $\varphi_{i}: \Lambda \rightarrow R$ and $c_{1}, \ldots, c_{n}$ are given real values. $\Lambda$ is the state space of the prices.

The solution of the problem is given by:

$$
\mathrm{g}\left(S_{T}\right)=\frac{\left[1-\frac{1-r}{r}\left(\sum_{i=1}^{n} \beta_{i}\left(c_{i}-\varphi_{i}\left(S_{T}\right)\right)\right)\right]^{\frac{1}{r-1}}}{\int_{-\infty}^{+\infty}\left[1-\frac{1-r}{r}\left(\sum_{i=1}^{n} \beta_{i}\left(c_{i}-\varphi_{i}\left(S_{T}\right)\right)\right)\right]^{\frac{1}{r-1}} d S_{T}}
$$

where $\beta_{1}, \ldots, \beta_{n}$ are to be obtained by $C-1$ and $C-2$.

Proof. We can proof above result by using calculus of variations for optimization of functionals (see Luenberger-1969 and Borwein-2003, Zellner and Highfield-1988). Therefore we can write the Lagrangian as:

$$
L=\frac{1}{1-r} \ln E^{g}\left(g^{r-1}\left(Y_{T}\right)\right)-\lambda\left(E^{g}\left[I_{\left\{-\infty<Y_{T}<\infty\right\}}\right]-1\right)-\sum_{i=1}^{n} \beta_{i}\left(E^{g}\left[\varphi_{i}\left(Y_{T}\right)\right]-c_{i}\right)
$$

Therefore we have:

$$
\frac{r}{r-1} \frac{g^{r-1}\left(S_{T}\right)}{E^{g}\left(g^{r-1}\left(Y_{T}\right)\right)}-\lambda-\sum_{i=1}^{n} \beta_{i} \varphi_{i}\left(S_{T}\right)=0
$$

where $\lambda$ is the Lagrange multiplier and taking expectation of last equation we get:

$$
\frac{r}{r-1} \frac{E^{g}\left(g^{r-1}\left(S_{T}\right)\right)}{E^{g}\left(g^{r-1}\left(Y_{T}\right)\right)}-\lambda\left(E^{g}\left[I_{\left\{-\infty<Y_{T}<\infty\right\}}\right]\right)-\sum_{i=1}^{n} \beta_{i} E^{g}\left(\varphi_{i}\left(Y_{T}\right)\right)=0
$$

Using given conditions we have:

$$
\frac{r}{r-1}-\lambda-\sum_{i=1}^{n} \beta_{i} c_{i}=0 \Leftrightarrow \frac{r}{r-1}-\sum_{i=1}^{n} \beta_{i} c_{i}=\lambda
$$

Now for value of $\lambda$ :

$$
\frac{1-r}{r} E^{g}\left(g^{r-1}\left(Y_{T}\right)\right)\left(\frac{r}{r-1}-\sum_{i=1}^{n} \beta_{i} c_{i}-\sum_{i=1}^{n} \beta_{i} \varphi_{i}\left(S_{T}\right)\right)^{\frac{1}{r-1}}
$$

Now we have:

$$
\begin{gathered}
\frac{r}{r-1} \frac{g^{r-1}\left(S_{T}\right)}{E^{g}\left(g^{r-1}\left(Y_{T}\right)\right)}-\lambda-\sum_{i=1}^{n} \beta_{i} \varphi_{i}\left(S_{T}\right)=0 \\
\frac{1-r}{r} E^{g}\left(g^{r-1}\left(Y_{T}\right)\right)\left(\lambda+\sum_{i=1}^{n} \beta_{i} \varphi_{i}\left(S_{T}\right)\right)=g^{r-1}\left(S_{T}\right)
\end{gathered}
$$




$$
g\left(S_{T}\right)=\frac{1-r}{r}\left(E^{g}\left(g^{r-1}\left(S_{T}\right)\right)\right)^{\frac{1}{r-1}}\left(1-\frac{1-r}{r}\left(\sum_{i=1}^{n} \beta_{i} c_{i}-\sum_{i=1}^{n} \beta_{i} \varphi_{i}\left(S_{T}\right)\right)\right)^{\frac{1}{r-1}}
$$

Now by using C-1 we get:

$$
1=\frac{1-r}{r}\left(E^{g}\left(g^{r-1}\left(Y_{T}\right)\right)\right)^{\frac{1}{r-1}} \int_{-\infty}^{+\infty}\left(1-\frac{1-r}{r}\left(\sum_{i=1}^{n} \beta_{i}\left(c_{i}-\varphi_{i}\left(S_{T}\right)\right)\right)\right)^{\frac{1}{r-1}} d S_{T}
$$

Therefore,

$$
\left(\int_{-\infty}^{+\infty} g^{r}\left(S_{T}\right) d S_{T}\right)^{\frac{1}{r-1}}=\left(\int_{-\infty}^{+\infty}\left(1-\frac{1-r}{r}\left(\sum_{i=1}^{n} \beta_{i}\left(c_{i}-\varphi_{i}\left(S_{T}\right)\right)\right)\right)^{\frac{1}{r-1}} d S_{T}\right)^{-1}
$$

Thus we obtain:

$$
\mathrm{g}\left(S_{T}\right)=\frac{\left[1-\frac{1-r}{r}\left(\sum_{i=1}^{n} \beta_{i}\left(c_{i}-\varphi_{i}\left(S_{T}\right)\right)\right)\right]^{\frac{1}{r-1}}}{\int_{-\infty}^{+\infty}\left[1-\frac{1-r}{r}\left(\sum_{i=1}^{n} \beta_{i}\left(c_{i}-\varphi_{i}\left(S_{T}\right)\right)\right)\right]^{\frac{1}{r-1}} d S_{T}}
$$

Theorem 2.4. Consider the weighted-Rényi entropy maximization problem

$$
\max H_{r}(g)=\frac{1}{1-r} \ln E^{g}\left(u\left(Y_{T}\right) g^{r-1}\left(Y_{T}\right)\right)
$$

Subject to

$$
\begin{array}{ll}
E^{g}\left[I_{\left\{-\infty<Y_{T}<\infty\right\}}\right]=1 & \mathrm{C}-1 \\
E^{g}\left[\varphi_{i}\left(Y_{T}\right)\right]=c_{i}, i=\overline{1, n}, & \mathrm{C}-2
\end{array}
$$

In particular $\varphi_{i}\left(S_{T}\right)=u\left(S_{T}\right), c_{1}=1$ then $E^{g}\left(u\left(S_{T}\right)\right)=1, \varphi_{i}: \Lambda \rightarrow R$ and $c_{1}, \ldots, c_{n}$ are given real values.

$\Lambda$ is the state space of prices and $u$ is a weighted function then solution of the problem is

$$
\mathrm{g}\left(S_{T}\right)=\frac{\left[\frac{1-\frac{1-r}{r}\left(\sum_{i=1}^{n} \beta_{i}\left(c_{i}-\varphi_{i}\left(S_{T}\right)\right)\right.}{u\left(S_{T}\right)}\right]^{\frac{1}{r-1}}}{\int_{-\infty}^{+\infty}\left[\frac{1-\frac{1-r}{r}\left(\sum_{i=1}^{n} \beta_{i}\left(c_{i}-\varphi_{i}\left(S_{T}\right)\right)\right)}{u\left(S_{T}\right)}\right]^{\frac{1}{r-1}} d S_{T}}
$$

where $\beta_{1}, \ldots, \beta_{n}$ are to be obtained by $C-1$ and $C-2$. 
The proof of theorem 2.4 is quite similar to the proof of theorem 2.3.

\subsubsection{Rényi Entropy Measure and EU-WE Framework}

Casquilho et al. [4] used EU-WE i.e. expected utility- weighted entropy framework under a 1parameter generalization of Shannon formula focused on an ecological and economic application at the landscape level. Following this approach the EU-WE framework, if $u$ is a positive utility application on $G$. In the next theorem we present a new result for the risk-neutral density using the frame of expected utility-weighted entropy for the case of Rényi-entropy maximization problem.

Theorem 2.5 Consider the case of Rényi-entropy maximization problem:

$$
\max H_{r}(g)=E^{g}\left(u\left(Y_{T}\right)\right)+\frac{1}{1-r} \ln E^{g}\left(u\left(Y_{T}\right) g^{r-1}\left(Y_{T}\right)\right)-\ln E^{g}\left(u\left(Y_{T}\right)\right)
$$

subject to the constraints $C-1$ and $C-2$ of theorem 2.4. Then solution of the problem is given by:

$$
\mathrm{g}\left(S_{T}\right)=\frac{\left[\frac{1}{r\left(1+\sum_{i=1}^{n} \beta_{i}+\lambda\right)}-\frac{1-r}{r}+\frac{1-r}{r} \frac{\left(\lambda+\sum_{i=1}^{n} \beta_{i} \varphi_{i}\left(S_{T}\right)\right)}{u\left(S_{T}\right)}\right]^{\frac{1}{r-1}}}{\int_{-\infty}^{+\infty}\left[\frac{1}{r\left(1+\sum_{i=1}^{n} \beta_{i}+\lambda\right)}-\frac{1-r}{r}+\frac{1-r}{r} \frac{\left(\lambda+\sum_{i=1}^{n} \beta_{i} \varphi_{i}\left(S_{T}\right)\right)}{u\left(S_{T}\right)}\right]^{\frac{1}{r-1}}} d S_{T}
$$

where $\beta_{1}, \ldots, \beta_{n}$ are to be obtained.

Proof. We can write the Lagrangian of the given problem:

$$
\begin{aligned}
L= & E^{g}\left(u\left(Y_{T}\right)\right)+\frac{1}{1-r} \ln E^{g}\left(u\left(Y_{T}\right) g^{r-1}\left(Y_{T}\right)\right)-\ln E^{g}\left(u\left(Y_{T}\right)\right)-\lambda\left(E^{g}\left[I_{\left\{-\infty<Y_{T}<\infty\right\}}\right]-1\right) \\
& -\sum_{i=1}^{n} \beta_{i}\left(E^{g}\left[\varphi_{i}\left(Y_{T}\right)\right]-c_{i}\right)
\end{aligned}
$$

i.e.,

$u\left(S_{T}\right)+\frac{r}{r-1} \frac{u\left(S_{T}\right) g^{r-1}\left(S_{T}\right)}{E^{g}\left(\left(u\left(Y_{T}\right)\right) g^{r-1}\left(Y_{T}\right)\right)}-\frac{1}{1-r} \frac{u\left(S_{T}\right)}{E^{g}\left(u\left(Y_{T}\right)\right)}-\lambda-\sum_{i=1}^{n} \beta_{i} \varphi_{i}\left(S_{T}\right)=0$

$E^{g}\left(u\left(Y_{T}\right)\right)+\frac{r}{r-1} \frac{E^{g}\left(u\left(Y_{T}\right)\right) g^{r-1}\left(Y_{T}\right)}{E^{g}\left(u\left(Y_{T}\right)\right) g^{r-1}\left(Y_{T}\right)}-\frac{1}{1-r} \frac{E^{g}\left(u\left(Y_{T}\right)\right)}{E^{g}\left(u\left(Y_{T}\right)\right)}-\lambda\left(E^{g}\left(I_{\left\{-\infty<Y_{T}<\infty\right\}}\right)\right)-\sum_{i=1}^{n} \beta_{i}\left(E^{g}\left(\varphi_{i}\left(Y_{T}\right)\right)\right)=0$

Now we get:

$$
E^{g}\left(u\left(Y_{T}\right)\right)-1-\lambda-\sum_{i=1}^{n} \beta_{i} \varphi_{i}\left(S_{T}\right)=0 \Leftrightarrow 1+\lambda+\sum_{i=1}^{n} \beta_{i} \varphi_{i}\left(S_{T}\right)=E^{g}\left(u\left(Y_{T}\right)\right)
$$


Using the following equation for the value of $\lambda$ :

$$
u\left(S_{T}\right)+\frac{r}{r-1} \frac{u\left(S_{T}\right) g^{r-1}\left(S_{T}\right)}{E^{g}\left(\left(u\left(Y_{T}\right)\right) g^{r-1}\left(Y_{T}\right)\right)}-\frac{1}{1-r} \frac{u\left(S_{T}\right)}{E^{g}\left(u\left(Y_{T}\right)\right)}-\lambda-\sum_{i=1}^{n} \beta_{i} \varphi_{i}\left(S_{T}\right)=0
$$

Let us put $\lambda+\sum_{i=1}^{n} \beta_{i} \varphi_{i}\left(S_{T}\right)=\gamma\left(S_{T}\right)$ then we get:

$$
\begin{aligned}
& g^{r-1}\left(S_{T}\right)=\frac{1-r}{r} \frac{E^{g}\left(u\left(Y_{T}\right) g^{r-1}\left(Y_{T}\right)\right)}{u\left(S_{T}\right)}\left[\frac{u\left(S_{T}\right)}{(1-r)\left(1+\gamma\left(S_{T}\right)\right)}-u\left(S_{T}\right)+\gamma\left(S_{T}\right)\right] \\
& g^{r-1}\left(S_{T}\right)=E^{g}\left(u\left(Y_{T}\right) g^{r-1}\left(Y_{T}\right)\right)\left[\frac{1}{r\left(1+\gamma\left(S_{T}\right)\right)}-\frac{1-r}{r}+\frac{1-r}{r} \frac{\gamma\left(S_{T}\right)}{u\left(S_{T}\right)}\right] \\
& g\left(S_{T}\right)=\left(E^{g}\left(u\left(Y_{T}\right) g^{r-1}\left(Y_{T}\right)\right)\right) \frac{1}{r-1}\left[\frac{1}{r\left(1+\gamma\left(S_{T}\right)\right)}-\frac{1-r}{r}+\frac{1-r}{r} \frac{\gamma\left(S_{T}\right)}{u\left(S_{T}\right)}\right] \frac{1}{r-1}
\end{aligned}
$$

Using C-1 we get:

$$
1=\left(E^{g}\left(u\left(Y_{T}\right) g^{r-1}\left(Y_{T}\right)\right)\right)^{\frac{1}{r-1}} \cdot \int_{-\infty}^{+\infty}\left[\frac{1}{r\left(1+\gamma\left(S_{T}\right)\right)}-\frac{1-r}{r}+\frac{1-r}{r} \frac{\gamma\left(S_{T}\right)}{u\left(S_{T}\right)}\right]^{\frac{1}{r-1}} d S_{T}
$$

i.e.,

$$
\left(E^{g}\left(u\left(Y_{T}\right) g^{r-1}\left(Y_{T}\right)\right)\right)^{\frac{1}{r-1}}=\left[\int_{-\infty}^{+\infty}\left[\frac{1}{r\left(1+\gamma\left(S_{T}\right)\right)}-\frac{1-r}{r}+\frac{1-r}{r} \frac{\gamma\left(S_{T}\right)}{u\left(S_{T}\right)}\right]^{\frac{1}{r-1}} d S_{T}\right]
$$

And we have obtained the result therefore the proof is complete.

\subsubsection{Pricing European Call and Put Options}

In this section we use the new risk neutral density function $g\left(S_{T}\right)$ obtained in the case of Rényi entropy, to evaluate the European call and put options on a dividend protected stocks. At time to expiry $T$, a call option pays $\max \left(0, S_{T}-K\right)$ and a put option pays $\max \left(0, K-S_{T}\right), K$ is the strike price. We use the linear pricing rule and the risk neutral density $g\left(S_{T}\right)$. Then the price of European Call can be written as follows: 


$$
\begin{aligned}
\text { Call } & =P E^{g}\left(\max \left(0, \mathrm{~S}_{T}-K\right)\right) \\
& =K \int_{K}^{\infty} g\left(S_{T}\right) S_{T} d S_{T}-P K \int_{K}^{\infty} g\left(S_{T}\right) d S_{T}
\end{aligned}
$$

which is equivalent to:

$$
\begin{aligned}
& \text { Call }=P\left(c_{1}-\int_{0}^{K} g\left(S_{T}\right) S_{T} d S_{T}\right)-P K\left(1-\int_{0}^{K} g\left(S_{T}\right) d S_{T}\right) \\
& \text { Call }=P c_{1}-P K G(K)-P K+P K(g(K)-0)+P \int_{0}^{K} G\left(S_{T}\right) d S_{T}
\end{aligned}
$$

We can use various kinds of risk neutral densities in the above given equation of Call to obtain $g(0)$. As we have presented in the some new results for the risk neutral densities, we can easily obtain the value of $g(0)$. Similarly we can write value of the Put option:

$$
\begin{aligned}
P u t & =P E^{g}\left(\max \left(0, K-\mathrm{S}_{T}\right)\right) \\
& =P \int_{0}^{K} g\left(S_{T}\right)\left(K-S_{T}\right) d S_{T} \\
& =P K G(K)-P \int_{0}^{K} g\left(S_{T}\right) S_{T} d S_{T}
\end{aligned}
$$

which is equivalent to:

$$
P u t=P \int_{0}^{K} G\left(S_{T}\right) d S_{T}
$$

\section{Conclusions}

In this article we have presented some approaches to obtain risk-neutral densities using three different types of entropy measures. We have used the famous entropy pricing theory of stock options. We have studied to price European call and put option using the underlying framework. The problem of extracting implied volatilities from market price of the options has always attained the concentration of researchers in option pricing but this is single statistic which can be extracted and depends on the option pricing model. The problem of getting risk-neutral density implies a comprehensive package without specifying any model has become crucial and entropy pricing theory is an alternative structure to solve such problems. The results are obtained by using the entropy measures that depend on the powers of the probability.

\section{Acknowledgments}

This work was supported by K.C Wong fund of the Ningbo University China. The authors express their gratitude to anonymous referees. 


\section{Author Contributions}

Each author contributed equally to the development of work.

\section{Conflicts of Interest}

The authors declare no conflict of interest.

\section{References}

1. Black,F.; Scholes, m. The pricing of options and corporate liabilities. Journal of Political Economy 1973, 81, 637-651.

2. Borwein, J.; Choksi, R.; Maréchal,P. Probability distributions of assets inferred from option prices via the principle of maximum entropy. Journal of Society for Industrial and Applied Mathematics 2003, 14, 464-478.

3. Buchen, P.W.; Kelly, M. The maximum entropy distribution of an asset inferred from option prices, Journal of Finance and Quantitative Analysis 1996, 31, 143-159.

4. Casquilho, J.; Neves, M; Rego, F. Extensos da funcao de Shannon e equilibrio de proporcoes-uma aplicacao ao mosaico de paisagem, Anais do Instituto Superior de Agronomia 1997, 46, 77-99.

5. Cover,T.M.; Thomas, J.A. Elements of information theory, $1^{\text {st }}$ ed.; Wiley: New York, 1991.

6. Droje, C.B.; Ian, R.C.B.; Irene, C.C. Option price calibration from Renyi entropy, Physics Letters A 2007, 366, 298-307.

7. Dedu, S.; Ciumara,R. Restricted optimal retention in Stop-Loss reinsurance under VaR and CTE risk measures, Proceedings of the Romanian Academy 2010, 11, 213-217.

8. Gulko, L. Dart boards and asset prices introducing the entropy pricing theory. Advance Econometrics 1997, 12, 237-276.

9. Gulko, L. The entropy theory of stock option pricing. International Journal of Theoretical and Applied Finance 1999, 2, 331-355.

10. Gulko, L. The entropy theory of bond option pricing. International Journal of Theoretical and Applied Finance 2002, 5, 355-383.

11. Guiasu, S. Weighted entropy, Reports on Mathematical Physics 1971, 2, 165-179.

12. Iosifescu, M. Sampling entropy for random homogeneous systems with complete connections, Annals of Mathematical Statistics 1965, 36, 1433-1436.

13. Jodra, P. Computer generation of random variables with Lindley or Poisson-Lindley distribution via the Lambert W function, Mathematics and Computers in Simulation 2010, 81, 851-859.

14. Guo, W.Y. Maximum entropy in option pricing: a convex-spline smoothing method. Journal of Futures Markets 2001, 21, 819-832.

15. Luenberger, D.G. Optimization by vector space methods; Wiley: New York, 1969.

16. Preda, V.; Balcau, C.; Entropy optimization with applications; The Publishing House of Romanian Academy, 2010.

17. Preda, V.; Dedu,S.; Sheraz,M.., New measure selection for Hunt-Devolder semi-Markov regime switching interest rate models, Physica A 2014,407,350-359. 
18. Preda, V; Dedu,S., Carmen, G. New class of Lorentz curves by maximizing Tsallis entropy under mean and Gini equality and inequality constraints, Physica A 2015, 436, 925-932.

19. Preda, V.; Sheraz, M. Risk neutral densities in entropy pricing theory of stock options using Lambert function and a new approach, Proceedings of the Romanian academy 2015, 16, 20-27.

20. Renyi, A. On measuring of entropy and information, In Proceeding of the 4th Barkely Symposium on Mathematics of Statistics and Probability 1961, 1, 547-561.

21. Rompolis, L.S. Retrieving risk neutral densities from European option prices based on the principle of maximum entropy, Journal of Empirical Finance 2010, 17, 918-937.

22. Shanon, C.E.; Weaver,W. The Mathematical theory of communication: The University of Illinois Press Urbana, 1964.

23. Shafee, F. Lambert function and a new non-extensive form of entropy, Journal of Applied Mathematics 2007, 72, 785-800.

24. Ubriaco, M.R. Entropies based on fractional calculus, Physics Letters A 2009, 373, 2516-2519.

25. Zhour, R.; Cai, R.; Tong, G. Application of entropy in finance: A review, Entropy 2013, 15, 4909-4931.

26. Zellner, A.; Highfield, R.A. Calculation of maximum entropy distributions and approximation of marginal posterior distributions, Journal of Econometrics 1988, 37, 195-209.

(C) 2015 by the authors; licensee MDPI, Basel, Switzerland. This article is an open access article distributed under the terms and conditions of the Creative Commons Attribution license (http://creativecommons.org/licenses/by/3.0/). 